\title{
Evaluación de almidones de arroz cubanos como adhesivo para la restauración de documentos: fuerza de adhesión y flexibilidad
}

\author{
Janet Diaz Navarro, Inés Baró Valle, Sofia Borrego
}

\begin{abstract}
Resumen: El uso de adhesivos vegetales en diferentes procesos de conservación es una tradición entre los restauradores de papel de origen oriental y de otros países. Los adhesivos de almidón son derivados de raíces y semillas tales como maíz, papa, yuca, arroz y trigo. Los más utilizados en conservación son los de trigo y arroz. En este trabajo se valora la conveniencia del uso de almidón de arroz procedente de variedades de plantas cubanas para tratamientos de restauración de obras sobre papel. Se evaluó la flexibilidad, fuerza de adhesión y estabilidad en el tiempo en probetas elaboradas de papel tratado con almidón de arroz. Dos muestras de almidón purificado a partir de arroz cubano con porcentajes intermedios de amilosa fueron comparadas con muestras de adhesivos comerciales (almidón de trigo, arroz y metilcelulosa). El uso de almidones de arroz cubanos para la restauración del patrimonio documental permite sustituir la importación de adhesivos que resultan extremadamente caros.
\end{abstract}

Palabras clave: almidón, adhesivo, arroz, papel, conservación

\section{Evaluation of Cuban rice starches for use in document conservation-restoration: adhesion strength and flexibility}

Abstract: The use of vegetable adhesives in different preservation processes is a tradition among oriental paper conservators and in other countries. Starch adhesives are derived from roots and seeds such as corn, potato, cassava, rice, and wheat. The most used in conservation are those of wheat and rice. In this work, the suitability of using rice starch from Cuban plant varieties for restoration treatments on paper's work is evaluated. Flexibility, adhesion strength, and stability over time were evaluated in samples made of rice starch-treated paper. Two samples of starch purified from Cuban rice with intermediate percentages of amylose were compared with samples of commercial adhesives (starch from wheat, rice and methylcellulose). The use of Cuban rice starches for the restoration of documentary heritage makes it possible to substitute the import of adhesives that are extremely expensive.

Keywords: starch, adhesive, rice, paper, preservation

\section{Avaliação dos amidos de arroz cubanos como adesivo para restauro de documentos: força de adesão e flexibilidade}

Resumo: A utilização de adesivos vegetais em diferentes processos de conservação é uma tradição entre os restauradores de papel orientais e de outros países. Os adesivos de amido são derivados de raízes e sementes tais como milho, batata, mandioca, arroz e trigo. Os mais comummente utilizados são os de trigo e arroz. Neste estudo avalia-se a adequação da utilização de amido de arroz de variedades vegetais cubanas para tratamentos de restauro de obras em papel. Avaliou-se a flexibilidade, força de adesão e estabilidade ao longo do tempo em amostras de papel tratado com amido de arroz. Duas amostras de amido purificado de arroz cubano com percentagens intermédias de amilose foram comparadas com amostras de adesivos comerciais (amido de trigo, amido de arroz e metilcelulose). A utilização de amidos de arroz cubanos para o restauro do património documental permite substituir a importação de adesivos extremamente caros.

Palavras-chave: amido, adesivo, arroz, papel, conservação 


\section{Introducción}

En la conservación de documentos y particularmente en los procesos de restauración, los adhesivos juegan un papel importante para el refuerzo de los materiales dañados, unir pedazos que estén rasgados o separados, como consolidantes, en la encuadernación, para la adhesión de pestañas, entre otros. Para que un adhesivo sea usado en la restauración y conservación de papel debe tener probadas características de estabilidad en el tiempo, ser reversible y con un adecuado grado de pureza, debe enlazarse bien a la superficie del papel sin provocar distorsiones planares por secado o reducción, además el método de aplicación no debe alterar su apariencia por cambio de color o manchas en el soporte (Group Wiki 2017; Lienardyy Van Damme 1989: 193). Numerosos son los adhesivos que han sido utilizados desde la antigüedad en la conservación y restauración de papel, el origen de los mismos ha sido variado como colas vegetales, colas animales y colas sintéticas (Zappala 1990: 34; Cannon 2011 a y b)

La calidad de los adhesivos vegetales ha sido reconocida, su uso es una tradición entre los restauradores de papel de origen oriental. Es bien conocido el adhesivo llamado Furunori utilizado en Japón desde la antigüedad que ha sido estudiado por diferentes autores desde el punto de vista químico y por sus propiedades como adhesivo (Hayakawa et al. 2007, 2002; Daniels 1988; Masuda 1984; Belard et al. 2009). Los adhesivos de almidón son derivados de raíces y semillas tales como maíz, papa, yuca, arroz y trigo (Horie 1987; Skeist, 1977). Los más utilizados en conservación son los de trigo y arroz. El origen del almidón y sus propiedades influyen en sus características como adhesivo. El adhesivo de almidón de arroz es generalmente considerado un adhesivo más débil que el de trigo y se usa en situaciones donde el trigo resulta demasiado fuerte (Villar y Martín 2010; Houwink y Salomon 1973).

El almidón es un carbohidrato de reserva de los vegetales que se obtiene de los granos de cereales, raíces y de los tubérculos, se diferencia de los demás hidratos de carbono presentes en la naturaleza por presentarse como un conjunto de gránulos (Whistler y Paschall 1965; French 1972: 8-25). Su estructura está representada por dos tipos de polímeros, ambos formados por monómeros de glucosa, que son la amilosa y la amilopectina. La amilosa es un polímero de unidades de D-glucosa, unidas por enlaces a-1,4 glucosídicos, esencialmente lineal, aunque muchas moléculas tienen unas pocas ramificaciones $a-1,6(0,3-$ $0,5 \%$ ). Las ramificaciones son o muy cortas o muy largas y están separadas por grandes distancias, permitiendo a la molécula actuar como un polímero lineal. Las cadenas glucosídicas de la amilosa se disponen en forma de hélice con 6 unidades de glucosa por vuelta y tiene un peso molecular comprendido entre150.000 y 1.000 .000 (Perez y Vergelati 1987: 141-148). La amilopectina es un polímero de D-glucosas, unidas por enlaces $a-1,4$, presenta ramificaciones con enlaces $\alpha-1,6(4-5 \%)$. Existe un punto de ramificación cada 15 - 30 restos de glucosa y el peso molecular es del orden de $10^{8}$ (Hizukuri 1986: 342-347).

La relación de las concentraciones de amilosa y amilopectina en cada tipo de almidón son responsables de la mayoría de sus propiedades. Se considera que el porciento de amilosa en el grano de trigo es mayor (18-27\%) que en el grano de arroz (17-19\%) (Group Wiki 2017), sin embargo el grano de arroz varía su contenido de amilosa desde 0,5 hasta un $33 \%$ de acuerdo a la variedad (Juliano, 2004: 41-85; Castillo 1981). El contenido de amilosa en las pastas de almidón de trigo envejecido con 2, 4 y 15 años varía en 19, 22 y $24 \%$ respectivamente (Daniels 1988).

El almidón es insoluble en agua pero a medida que asciende la temperatura el gránulo de almidón se hincha hasta la ruptura de su estructura natural, a la temperatura que alcance este estado se le llama temperatura de gelatinización, una pasta de almidón es una masa viscosa consistente de amilosa y amilopectina. Si una solución de almidón concentrada se enfría, en ausencia de agitación, se forma un gel rígido. Este retorno al estado insoluble se llama retrogradación. La retrogradación del almidón cocido involucra a los dos polímeros constituyentes, la amilosa y amilopectina. La retrogradación de la amilosa es mucho más rápida que la de la amilopectina y en el caso del almidón de trigo es mayor por tener fracciones más largas de sus polímeros (Castillo 1981; Horie 1987; Skeist 1977). Las películas secas de estos polisacáridos son incoloras y permite su uso en los procesos de consolidación.

Se conservan más de 115000 accesiones de arroz en el banco de germoplasma del Instituto Internacional de Investigaciones del Arroz (IRRI) de Filipinas (Castillo 2016). En Cuba se ha logrado genéticamente, a través de los métodos de cruzamientos y de mutaciones, más de 80 nuevas variedades de arroz con diferentes propiedades evaluadas fundamentalmente para el consumo de la población (Suarez et al. 2010).En este trabajo se estudia el almidón de arroz de dos variedades cubanas, con un contenido de amilosa intermedio (21-25\%) (Juliano 2004). Se evalúa la adaptabilidad y conveniencia de estos almidones para tratamientos de restauración en documentos sobre papel y su estabilidad en el tiempo. Se comparan con el almidón de trigo, de arroz, y la metilcelulosa comerciales para restauración de papel, en cuanto a fuerza de adhesión y flexibilidad (entre los almidones). La importación de adhesivos para la restauración documental resulta extremadamente cara, por lo que sustituir parte de éstos por almidón de arroz de procedencia nacional es importante para la salvaguarda del patrimonio documental cubano.

\section{Materiales y métodos}

\section{- Características de los adhesivos utilizados en el estudio}

Se emplearon dos muestras de almidón de arroz que se obtuvieron en el Instituto de Granos de Cuba a partir de 
dos variedades de arroz cubanas. Se denominaron almidón de arroz cubano AAC1 y AAC2, las cuales se describen por Hernández (2003:1001-1006) y se caracterizan por tener un alto grado de pureza determinada por microscopía electrónica, 22.72 y $22.39 \%$ de amilosa y 77.28 y 77.61 de amilopectina respectivamente, con rango de temperatura de gelatinización entre $74-80^{\circ} \mathrm{C}$. La selección de estas variedades está relacionado con el porcentaje de amilosa que evidencia un porcentaje mayor que el que presentan los adhesivos de almidones de arroz comerciales y se asemejan al por ciento de amilosa de los adhesivos de almidón de trigo (Group Wiki 2017). Como muestras de referencia se utilizaron adhesivos comerciales para restauración y fueron almidón de arroz de la marca Bresciani, con un 19\% de amilosa; almidón de trigo de la marca Gaylor (no se reporta específicamente su por ciento de amilosa); y metilcelulosa de la marca TALAS (viscosidad 2000 cPs al $2 \%$ en agua) por ser uno de los adhesivos más utilizados en nuestro laboratorio y haber sido usado en otros estudios como control en la comparación con otros almidones (Pataki 2009; Da Silva et al. 2018).

\section{-Preparación de las pastas de almidón y de metilcelulosa}

Numerosas son las recetas para la preparación de pasta de almidón descritas en la literatura en general, seis de ellas están resumidas en la monografía "Adhesives for paper" (Group Wiki 2017) incluyendo las utilizadas en la Biblioteca del Congreso y en el Museo de la Universidad de Harvard. La manera de preparar la pasta influye en sus propiedades, es necesario obtener una pasta sin aglomerados, homogénea y de fácil manipulación. En nuestro caso se probaron 3 recetas para evaluar la consistencia y manejo. Seleccionamos el procedimiento que describimos a continuación porque se obtiene una pasta adecuada y resulta fácil de realizar. Se colocaron $5 \mathrm{~g}$ de polvo de almidón en un vaso de precipitado y se añadieron $25 \mathrm{ml}$ de agua se agitó y se dejó reposar por una hora; a esta mezcla se añadió a $25 \mathrm{ml}$ de agua a ebullición y se agitó de 5 a 10 minutos manteniendo la mezcla en la plancha de calentamiento. Luego se separó del calor y se dejó enfriar. Este proceso de formación de la pasta de almidón se realizó para las 2 muestras en estudio y para los controles. Las muestras de almidón de arroz formaron una pasta no grumosa y fácilmente tratable [Figura 1].

La metilcelulosa se preparó añadiendo a $5 \mathrm{~g}$ del polvo, 25 $\mathrm{ml}$ de agua y agitando con una varilla de vidrio, se dejó reposar 15 minutos. Luego se fue añadiendo y agitando de la misma forma hasta completar $150 \mathrm{ml}$ de agua y así la pasta quedó con una consistencia suave y adecuada.

\section{- Determinación del peso añadido por el almidón y su correlación con la flexibilidad}

Para conocer el peso añadido de cada almidón al papel y correlacionarlo con la flexibilidad, se elaboraron probetas a partir de papeles de manufactura antigua, fabricados entre 1892 y 1893 . Estos folios no presentaban escrituras, pero en algunos casos poseían manchas de tinta o sombras de tintas ferrogálicas que habían migrado. Fueron elaborados con pulpa mecánica y tienen un buen estado de conservación, contaban con un pH entre 4 y 5, no mostraban fragilidad, tenían color amarillo por del envejecimiento característico del papel antiguo. Se tomaron 5 folios y se les realizó una limpieza húmeda en una solución de hidróxido de calcio para su desacidificación con la intensión de que su $\mathrm{pH}$ subiera entre 6 y 7. La limpieza de los folios extraídos de reservas de materiales antiguos es necesaria al igual que la que se le realiza a los materiales originales previo a cualquier tratamiento de restauración. Esta elimina cualquier suciedad que pueda interferir en los resultados, además de estabilizar las fibras con la disminución de la acidez del papel. 25 tiras de papel de $5 \times 2 \mathrm{~cm}$ fueron destinadas para cada tipo de almidón, y fueron pesadas en una balanza analítica antes del tratamiento. Sobre ellas se extendió una capa homogénea de almidón con brocha lo más uniforme posible, que se dejó secar a temperatura ambiente. Luego, se pesaron nuevamente para calcular el peso añadido por el adhesivo. Con este ensayo se pretendió correlacionar
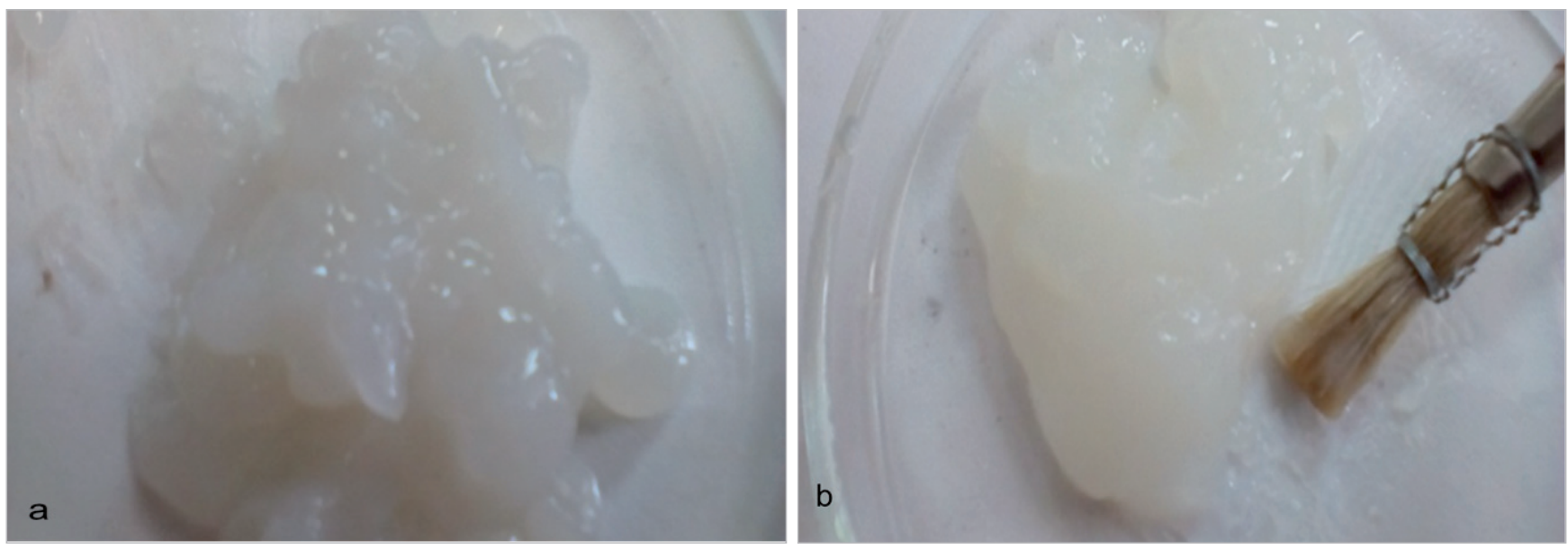

Figura 1.-Muestra la consistencia de las pastas de almidón aplicadas a las probetas. a) AAC1, b) AAC2. 
el peso añadido de cada uno de los almidones con la flexibilidad de las muestras determinada de forma manual por los restauradores a través de la apreciación al tacto de su rigidez.

- Fuerza de adhesión de los adhesivos de almidón y la metilcelulosa

Para la medición de la fuerza de adhesión se realizó el ensayo de pelado en T a 180 grados (Hayakawa et al. 2007; Caulfield y Gunderon 1990; Rampton 2005). Esta prueba determina la resistencia del adhesivo al aplicar una fuerza para separar una unión encolada. Para este ensayo se necesitan papeles que tengan una buena resistencia a la tracción y que no se rompan antes de que se culmine el ensayo. Aunque generalmente se utiliza papel, otros autores utilizan materiales sintéticos (Rampton 2005). Para nuestras probetas, utilizamos papel para impresoras de recibos, que son resistentes a las tensiones del mecanismo de las máquinas donde son colocados. Se hicieron 5 probetas por cada adhesivo respetando el número y las dimensiones sugeridas por las normas para este tipo de ensayo (AENOR 2005). Cada probeta midió $2,5 \mathrm{~cm}$ de ancho por $20 \mathrm{~cm}$ de largo y fueron pegadas solo hasta los $15 \mathrm{~cm}$ de largo dejando $5 \mathrm{~cm}$ sin encolar para permitir que se sujeten por las mordazas del equipo y se aplique la tensión sobre el papel pegado. Los adhesivos fueron aplicados con brocha de forma homogénea, tratando de lograr la mayor uniformidad posible, evitando las arrugas y los dobleces. Las muestras se secaron en bandejas en condiciones ambientales del laboratorio a temperatura de $27^{\circ} \mathrm{C}$ y humedad relativa de $45 \%$. Los ensayos se realizaron con la torre INSTROM modelo 3345, que recoge un gráfico de los valores de resistencia que presentan los adhesivos al separarlos de sus soportes.

\section{— Estudio de la estabilidad del almidón en el tiempo}

Con el objetivo de comprobar la eficacia de los adhesivos de almidón de arroz y poder utilizarlos en la restauración de documentos, en el año 2011 se laminaron papeles de manufactura antigua (como los descritos para el ensayo “Determinación del peso añadido por el almidón y su correlación con la flexibilidad") luego de su limpieza y desacidificación. Estos se almacenaron en uno de los depósitos del Archivo Nacional de Cuba (ARNAC) en las mismas condiciones ambientales que el resto de los documentos que en él se encontraban (Temperatura promedio $=27^{\circ} \mathrm{C}$, Humedad relativa promedio $=65 \%$ ) y oscuridad. Estos parámetros están descritos en el estudio de Vivar et al. (2016). Entre los almidones de arroz utilizados provenientes de diferentes variedades de arroz de origen cubano, se encontraban AAC1 y AAC2 que son objeto de los ensayos descritos en este artículo. Estas probetas se examinaron a simple vista y con el microscopio manual de 75X para observar la superficie del papel almidonado y se comprobó su estado general de conservación, luego de un envejecimiento natural por 8 años. Además fueron comparadas con las muestras consolidadas de solo 1 día.

\section{- Análisis estadístico de los datos}

Se utilizó el programa Statgraphics plus 5 para el análisis estadístico de los datos obtenidos. La prueba de KruskalWallis, permitió comparar los papeles con y sin almidón, así como los almidones cubanos con los controles comerciales. A través de un análisis de varianza (ANOVA) complementado con un análisis de rangos múltiples mediante el método de diferencia de mínimos cuadrados (DMC o LSD, siglas en inglés) para un $95 \%$ de confianza se comparó la fuerza de adhesión de los papeles tratados con los diferentes adhesivos.

\section{Resultados y discusión}

\section{- Peso añadido por los adhesivos al papel y su relación con la flexibilidad}

La flexibilidad es la elongación máxima que puede soportar un material, sin llegar a romperse, sometido a fuerzas de tracción o flexión. Este concepto está ligado al concepto de elasticidad, que es la facultad de elongación y orientación de las cadenas intermoleculares en la dirección de la fuerza ejercida sobre ellas (Gómez 2008).

La flexibilidad en los procesos de laminación y consolidación está relacionada con el adhesivo. La flexibilidad de los adhesivos es un factor a tener en cuenta en la conservación, manipulación y almacenamiento. Es importante en los procesos de restauración, por las tensiones que se pueden crear en los documentos constituyendo un factor en su deterioro. En el montaje de rollos japoneses, compuestos por diferentes capas de papel se utiliza el Furunori (almidón de trigo fermentado). Este se considera menos fuerte que el almidón de trigo sin envejecer (Shinnori) y con una viscosidad más baja lo que le confiere una mayor flexibilidad (Belard et al. 2009). Otro ejemplo es la aplicación por los restauradores de mezclas de almidón de trigo con metilcelulosa por la flexibilidad que le aporta este adhesivo a la mezcla (Villar y Martín 2010; Baker 1990; Quandt 2002; Wagner 1996; Brückle 1996).

La formación de la película seca está relacionada con las propiedades de la amilosa y la amilopectina. La amilosa posee una estructura lineal regular por lo que forma una película fuerte, en cambio la amilopectina como tiene una estructura más amorfa, forma películas más débiles (Horie 1987). Pataki (2009) midió la flexibilidad de forma empírica a través de símbolos luego de la apreciación de movimientos de las muestras con las manos. Para realizar pruebas de flexión en un punto de las películas de diferentes adhesivos Flores (2016) utilizó un MICROTEST (marca Deben - Gatan) con conexión a una ordenador para almacenar los datos. En nuestro estudio se determinó cualitativamente la 
flexibilidad que aportan los adhesivos al papel de forma manual (a través del tacto) por los restauradores.

En la evaluación del efecto del almidón sobre los papeles se compararon los pesos de las probetas controles (sin almidón) con las tratadas, se observó que no existió una distribución normal entre los datos, por lo que fue necesario utilizar la prueba Kruskal-Wallis que demostró que no existieron diferencias significativas entre estos papeles. Este es un resultado esperado que evidencia que la laminación con almidón no aporta un peso significativamente mayor al papel [Figura 2].

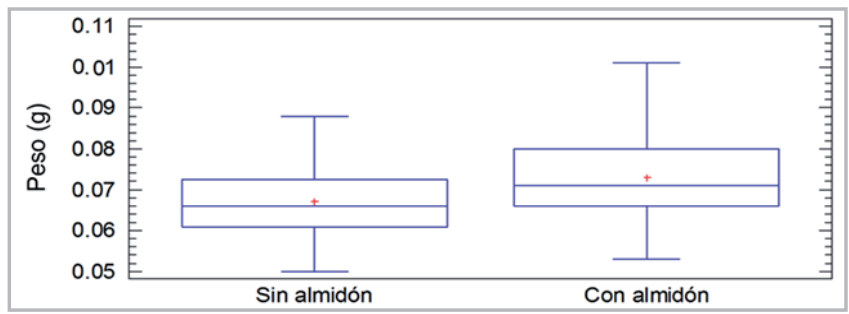

Figura 2.- Valores de las medias y las desviaciones estándares de los pesos de las probetas con y sin almidón según la prueba Kruskal-Wallis para un 95\% de confianza.

La tabla 1 muestra la comparación de los pesos añadidos por los diferentes adhesivos. Como se observa no existen diferencias significativas entre los almidones de arroz cubanos y el almidón de Bresciani. El almidón de trigo solo presenta diferencias significativas con $A A C 2$, siendo estadísticamente no significativo con los almidones de arroz Bresciani y AAC1.

\begin{tabular}{|c|c|}
\hline Almidones & Peso añadido \pm DE \\
\hline Bresciani & $0.072 \pm 0.010 \mathrm{ab}$ \\
\hline Trigo & $0.069 \pm 0.011 \mathrm{a}$ \\
\hline AAC1 & $0.074 \pm 0.012 \mathrm{ab}$ \\
\hline AAC2 & $0.076 \pm 0.014 \mathrm{~b}$ \\
\hline
\end{tabular}

$\mathrm{n}=25$. DE: Desviación estándar. a, b, ab: Indican diferencias significativas o no para un $95 \%$ de confianza $(p \leq 0.05)$

Tabla 1.- Resultado del peso añadido por el almidón a los papeles.

A cada muestra de almidones se le evaluó la flexibilidad por varios restauradores por apreciación al tacto. No fueron encontradas diferencias entre la AAC1 y el Bresciani. El trigo se encontró un poco más flexible por algunos mientras que el AAC2 coincidió para todos como el que aportaba más firmeza al papel. Al comparar estos resultados del peso añadido con el análisis estadístico realizado se evidenció total coherencia entre ellos, lo que confirma que el peso añadido por los almidones de arroz que se analizaron en este trabajo no afectó la flexibilidad de los papeles, aunque el AAC2 aporta una mayor consistencia. Estos resultados demuestran la posibilidad de usar los almidones AAC1 y ACC2 en la consolidación de los materiales de obra gráfica y profundizar en su estudio. Los almidones presentan estructuras químicas complejas que hay que tener en cuenta ya que influyen en sus propiedades (Skeist 1977; Whistler y Paschall 1965).

\section{— Estabilidad y preservación de las probetas de almidón}

El estudio de los procesos de envejecimiento en cámaras artificiales es uno de los recursos que han usado los investigadores para la prueba de eficacia en diferentes materiales sometiendo las muestras a condiciones extremas de temperatura, humedad relativa y radiación UV por un tiempo determinado (Bansa 2002). El envejecimiento natural requiere de mucho tiempo por lo que es impracticable y no está contemplado que tiempo necesita un material para probar su eficacia (Horie 2010). En estudios previos el almidón de trigo y arroz fueron usados como adhesivos en restauración, donde se sometieron a condiciones extremas de temperatura y humedad relativa y sus parámetros físico químicos se han mantenido bastante estables (Da Silva et al. 2018). En nuestro país el uso de almidones como adhesivos para la restauración en papel se limitó hasta casi desaparecer por las condiciones ambientales características de un clima tropical húmedo donde la mayor parte del año la humedad relativa supera el $60 \%$ y temperatura gira entorno a los $30^{\circ} \mathrm{C}$. Este ambiente propicia el crecimiento de microorganismos que degradan el almidón por lo que es un factor que hay que tener en cuenta en la conservación de los documentos sobre papel. No obstante, desde el año 2011 se valoró en Cuba la posibilidad de usar el almidón por sus propiedades adhesivas, ya que su utilización es frecuente a nivel internacional y además se tuvo en cuenta que en Cuba se pueden obtener almidones de alta pureza a partir de variedades de arroz nacionales. Por este motivo, los almidones AAC1 y AAC2 se comenzaron a ensayar y se han usado en este estudio por haber sido envejecidos de forma natural.

En este trabajo mostramos el análisis visual y al microscopio 75X de las muestras laminadas con los almidones AAC1 y AAC2 que han permanecido conservados en los depósitos del ARNAC por 8 años; aunque no es un tiempo prolongado, nos permite monitorear el comportamiento de esos materiales en las condiciones ambientales en las que se conservan nuestros documentos. Estas muestras no presentan ningún deterioro relacionado con fragilidad o pérdida de material, mantienen una buena flexibilidad, no hay señales de ataques de insectos o microorganismos y no se observaron deformaciones en el soporte ni cambio de coloración a la luz natural [Figura 3].

Al microscopio 75X se observó una estabilidad de la capa de almidón y no hay diferencias con las muestras tratadas de un día [Figura 4]. Estos resultados son los esperados ya que la literatura describe a los almidones como los adhesivos que desarrollan una mayor estabilidad en el tiempo (Smith et al. 1989; Da Silva et al. 2018). 

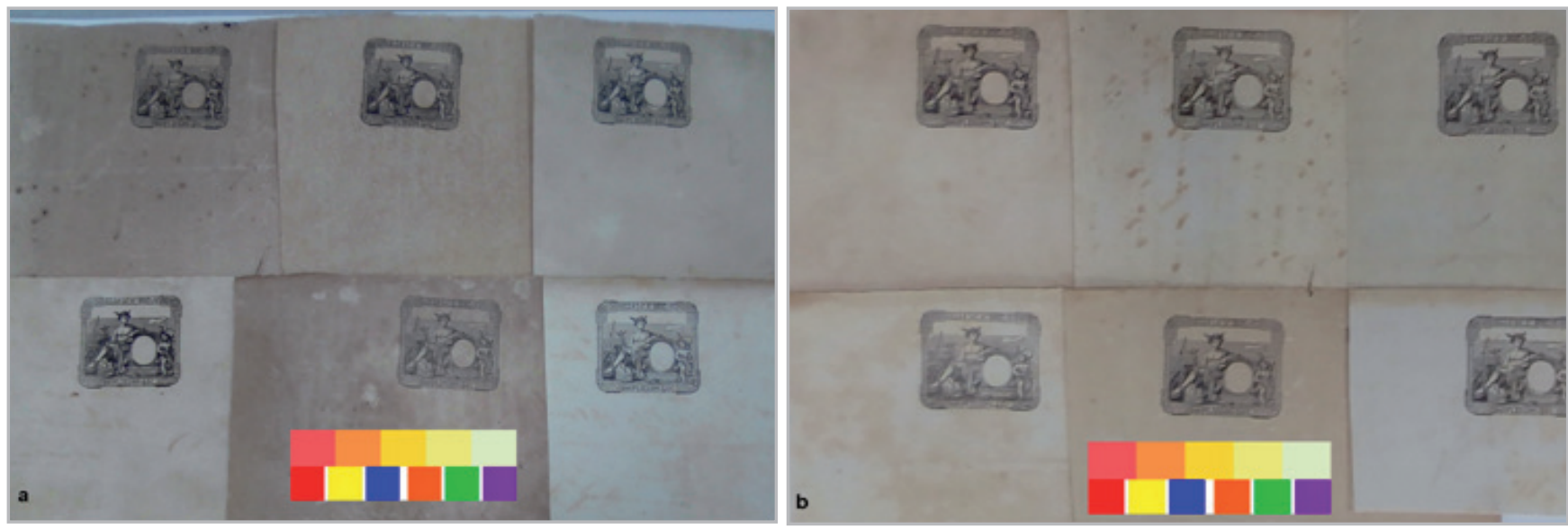

Figura 3.- Muestras de folios sin consolidar (a) y consolidadas (b) con almidón de arroz envejecidas por 8 años

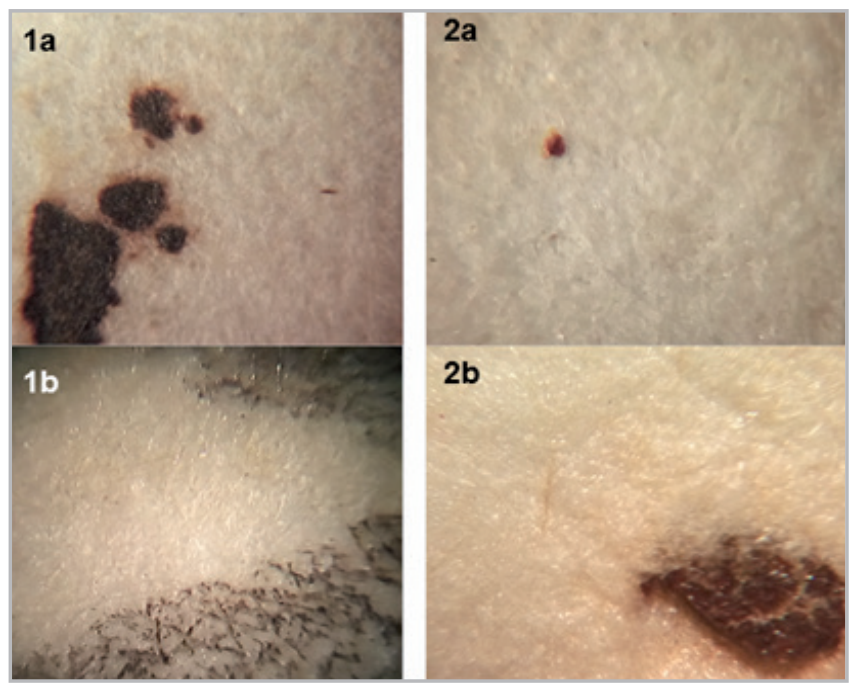

Figura 4.- Probetas bajo el microscopio 75X. 1a y 2a son AAC1 y AAC2 envejecidas por ocho años, $1 b$ y $2 b$ son AAC1 y AAC2 con 1 día de aplicada la pasta de almidón.

\section{- Fuerza de adhesión}

La adhesividad o adherencia es un conjunto de fuerzas de atracción que pueden ejercerse entre dos superficies cuando estas están en contacto (Houwinks y Salomón 1978). Los adhesivos son sustancias que garantizan la adhesión entre dos partes, una superficie sólida y una segunda fase que puede ser sólida o líquida formando lo que se denomina unión adhesiva. La unión adhesiva depende de varios factores, como son la humectación, rugosidad, estado superficial, deslizamiento del adhesivo y las fuerzas existentes entre el adhesivo y el sustrato (Flores 2016).

Los almidones son adhesivos vegetales reconocidos desde el principio de la historia (Group Wiki 2017; Herrick 2005). Se aplican sobre las superficies por medio de emulsiones o disoluciones, unido a una humectación del soporte que facilita la apertura de los poros del papel y la penetración del adhesivo, luego la evaporación del solvente da lugar a una película sólida adherida al soporte. No hay una teoría que explique el fenómeno de adhesión en su conjunto, diferentes modelos explican cada caso en particular. La atracción de una superficie por otra es llamada adhesión específica, es mayor entre los materiales que son similares químicamente como es en el caso del almidón y la celulosa teniendo en cuenta que ambos son polímeros de glucosa. Entre ellos se involucran fuerzas intermoleculares como de Van Der Waals y enlaces por puentes de hidrógeno, lo que los autores Ilaman teoría de quimiadsorción o teoría de adsorción termodinámica (Martín y Orgilés 1992).

Para la medición de la fuerza de adhesión de los diferentes adhesivos se realizó el ensayo de pelado en T a 180 grados (Hayakawa et al. 2007; Caulfield y Gunderon 1990; Rampton 2005). Cada probeta se somete a una fuerza para la separación de la unión adhesiva obteniendo la resistencia al pelado $(\mathrm{N} / \mathrm{mm})$. Los resultados procesados estadísticamente se muestran en la Figura 5.

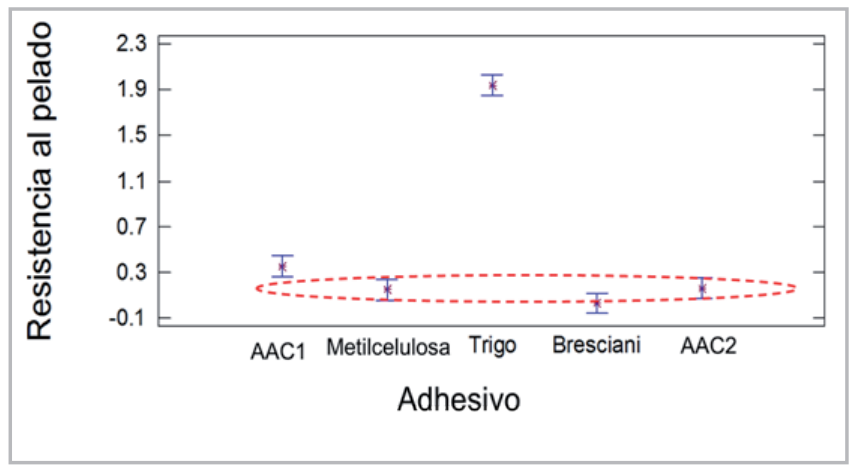

Figura 5.- Resistencia al pelado en $T$ para las muestras y los controles. Se muestran las medias y las desviaciones estándares según el ANOVA complementado con un análisis de rangos múltiples mediante el método de LSD.

En las probetas de los almidones AAC1, y AAC2, trigo y metilcelulosa se ocasionó un desgarre y ruptura del papel. Sin embargo, en las probetas tratadas con el almidón de arroz Bresciani fue posible separar por completo las tiras de papel por lo que manifiestan una menor fuerza adhesiva, mediante el ensayo de pelado en T. 
El almidón de trigo posee la mayor fuerza de adhesión, y se demostró que es significativamente alta, comportamiento que está en correspondencia con lo descrito en la literatura (Group Wiki 2017). Según el ANOVA no hay diferencias estadísticamente significativas entre el almidón de arroz de Bresciani, AAC2 y la metilcelulosa, que como se observa en la Figura 5 forman un grupo homogéneo, mientras que el almidón AAC1 se diferencia significativamente del resto de los adhesivos con una fuerza de adhesión superior, aunque muy por debajo del almidón de trigo. Este resultado puede estar relacionado con el por ciento de amilosa presente en este almidón. La afinidad de la amilosa por las sustancias polares como la celulosa, permite que se adsorba selectivamente (Pacsu y Mullen 1941). La fuerza de adhesión de los almidones depende en gran medida de su fuente y su composición química, en este caso el AAC1 supera la fuerza de adhesión de AAC2 teniendo valores de amilosa semejantes, demostrándose así que los factores estructurales característicos de cada variedad de almidón influyen también en su poder de adhesión.

\section{Conclusiones}

El uso de los almidones en climas tropicales fue descartado en muchos de los laboratorios de restauración, ya que se consideran vulnerables a los procesos de deterioro por las condiciones ambientales. Sin embargo, si el almidón presenta una alta pureza, como los usados en este estudio, y se aplica sobre materiales limpios de restos de suciedad y sin contaminación, se pueden usar en los procesos restaurativos ya que se ha demostrado que pueden tener una gran estabilidad en el tiempo a pesar de las condiciones climáticas naturales, características de ambientes tropicales. Esto permite garantizar su utilización, reduciendo el uso de los adhesivos importados y de mayor costo.

Se demostró estadísticamente que la consolidación del papel con las variantes de almidón evaluados no aportaron un peso significativo al papel. En este punto es necesario tener en cuenta que la aplicación del adhesivo debe ser en una capa homogénea y fina sobre el papel húmedo, consiguiendo que el almidón aporte una estabilización con la menor cantidad de adhesivo posible. Las muestras tratadas con el almidón AAC2 presentaron un ligero aumento en la firmeza del papel por apreciación al tacto de los restauradores.

Los almidones AAC1 y AAC2 presentaron diferencias estadísticamente significativas en cuanto la fuerza de adhesión; teniendo en cuenta estas características se puede implementar su uso en dependencia de la obra que se va restaurar y el tratamiento a emplear. El fenómeno de adhesión tiene mecanismos complejos donde intervienen diferentes factores. El por ciento de amilosa es un elemento a considerar, pero no es una guía exclusiva para determinar la fuerza de adhesión. Hay que tener en cuenta otras propiedades como son el grado de polimerización y la retrogradación de los almidones.

\section{Agradecimientos}

Agradecemos al Instituto de Granos de Cuba, en especial al Dr. Dámaso Castillo por el interés en la realización de este trabajo y el suministro de muestras de almidón de variedades cubanas de arroz. También agradecemos a la Dra. Gemma Contreras y al Dr. Livio Ferraza del Instituto Valenciano de Conservación, Restauración e Investigación por la realización del ensayo de pelado en $\mathrm{T}$ a $180^{\circ}$ a las probetas de papel en el Laboratorio de Materiales de este instituto y a la Dra. Matilde Anaya por el procesamiento estadístico de los datos.

\section{Referencias}

AENOR (2005). "Adhesivos para papel y cartón, envases y embalajes. Ensayo de pelado en " $\mathrm{T}$ " a $180^{\circ}$ para una unión encolada de adherente flexible sobre flexible". UNE-EN 11339, Madrid.

BANSA, H. (2002). "Accelerated ageing of paper: Some ideas on its practical benefit". Restaurator, 23: 106-117. https://doi. org/10.1515/REST.2002.106

BELARD, R., HIGUCHI, H., PERRY, J. (2009) “Furunori (aged wheat starch paste): Challenges of production in non-traditional settings". Journal of the Institute of Conservation, 32(1): 31-51. https://doi.org/10.1080/19455220802630735

BRÜCKLE, I. (1996). "Update: remoistenable lining with methylcellulose adhesive preparation". The Book and Paper Group Annual, 15: 25 - 26.

CANNON, A. (2011). "Adhesives used for mounting photographic prints of the 19th and early 20th centuries" AICCM Bulletin, 32: 1-12.

CANNON, A. (2011) “Interactions between adhesives from natural sources and paper substrates". En Symposium - Adhesives and Consolidants for Conservation: Research and Applications, Ottawa: Canadian Conservation Institute, 1-16.

CASTILLO, D. (1981). "El grano de arroz: Características fisicoquímicas de sus almidones relacionadas con las propiedades mecánicas y de cocción", Centro de Información y Divulgación Agropecuario. Minag, 1: 23-26.

CASTILLO, D. (2016). Protagonismo del agua, y de las proteínas, en la arquitectura botánica y la calidad del grano de arroz. La Habana: Universidad de La Habana, Tesis para optar por el título de Doctor en Ciencias.

CAULFIELD, D.F., GUNDERSON, D.E. (1990). Paper testing and strength characteristics. Testing and monitoring of paper aging, Edited by Philip Luner. Tappi Press: 43-52. 
DANIELS, V. (1988). "A Study of the Properties of Aged Starch Paste (Furunori)", En The Conservation of Far Eastern Art, ed. J.S. Mills, P. Smith, and K. Yamasaki. London: International Institute for Conservation. 5-10. https://doi.org/10.1179/sic.1988.33.1.5

DA SILVA, I., CASIMIRO, M.H., MACEDO, M., OLIVEIRA, S. (2018). "Adhesives used in paper conservation: Chemical stability and fungal bioreceptivity". Journal of Cultural Heritage, 34: 53-60. https://doi.org/10.17632/4m3f7bjct2.1

FLORES, L. (2016). Estudio del comportamiento de adhesivos sintéticos y de sus propiedades físico-químicas aplicados sobre soporte lígneo. Universitat Politècnica de València, Tesis de Máster de Conservación y Restauración de Bienes Culturales, España.

FRENCH, D. (1972). "Fine structure and relationship to the organization of starch granules". Journal of Japanese Society of Starch Science, 19(1): $\underline{\text { https://doi.org/8-25.10.5458/JAG1972.19.8 }}$

GÓMEZ, M.L. (2008). La Restauración: examen científico aplicado a la conservación de obras de arte. Madrid: Instituto del Patrimonio Histórico Español ed., $5^{\text {a }}$ ed.

GROUP WIKI BOOK AND PAPER (2017). "Adhesives for Paper", http://www.conservation-wiki.com/wiki/adhesivesforpaper. [consulta: 16/8/2018]

HAYAKAWA, N., KIGAWA, R., NISHIMOTO, T., SAKAMOTO, K., FUKUDA, S., KIMISHIMA, T., OKA, I., KAWANOBE, W. (2007). "Characterization of Furunori (aged paste) and preparation of a polysaccharide similar to Furunori". Studies in Conservation, 52: 221-32. https://doi.org/10.1179/sic.2007.52.3.221

HAYAKAWA, N., KIGAWA, R., KAWANOBE,W. HIGUCHI, Y., OKA I. (2002). "Basic research of aged paste (Furunori) traditional japanese restoration material by GPC and HPLC for organic acid analysis". Science for Conservation, 41: 15-28.

HERNÁNDEZ, A.A., CASTILLO, D., BENEDITO, C., DUFFAY, I.H. (2003). "Calidad del grano de arroz interpretada a través de las propiedades del almidón". Revista Cubana de Farmacia, 37: 10011006.

HIZUKURI, S. (1986) "Polymodal distribution of the chain length of amylopectin, and its significant". Carbohydrate Research, 147(2): 342-347. https://doi.org/10.1016/S0008-6215(00)90643$\underline{8}$

HORIE, C.V. (1987). "Materials for conservation: organic consolidants, adhesives, coatings". London, Batterworths and Co.:135-136.

HOUWINK, R., SALOMON, G. (1973). Adherencia y adhesivos. Enciclopedia de la Química Industrial. Urmo, Bilbao.

JULIANO, B.O. (2004). Overview on rice. Encyclopedia of Grain Science. First edition. Editors Wrigley C., Corke H. y Walker C. E. Editorial Elsevier. Academic Press. New York and London: 41-85.
LIENARDY, A., VAN DAMME, P. (1989) Manuel de Conservation et de restauration du Napier. Ed. Institut Royal du Patrimoine Artistique, Bruxelles: 193.

MARTIN, J.M., ORGILES, A.C. (1992). Adhesión. Tecnología y Fundamentos. Editorial: INESCOP \& Universidad de Alicante, España.

MASUDA, K. (1984). "Vegetable adhesives used in the workshop of the Hyōgushi, restorer and mounter of japanese paintings in adhesives and consolidants", En Vegetable Adhesives, ed. N.S. Brommelle London: International Institute for Conservation: 127-8.

PACSU, E. y MULLEN, J. W. (1941). "Separation of starch into its two constituents". Journal of the American Chemical Society, 63(4): 1168-1169.

PEREZ, S., VERGELATI, C. (1987). “Solid State and Solution Features of Amylose and Amylose Fragments". Polymer Bulletin, 17(2): 141148. https://doi.org/10.1007/BF00256880

RAMPTON, A. (2005). “Almidón de casaba y almidón de trigo: Estudio comparativo para su uso en conservación de papel". En: II Congreso del Grupo Español del IIC, Barcelona, 42-48.

SKEIST, I. (1977). Handbook of Adhesives. Second Edition.Van Nostrand Reinhold Company.

SUAREZ, E., DEUS, J.E., GONZALES, F., YSHIRAISHI M. (2010) Manual de producción de semillas para el arroz popular. Instituto de Investigaciones de Granos de Cuba, y Agencia de Cooperación Internacional (JICA), Japón, 48

VILLAR, L., MARTíN, S. (2010). “Estudio formal y compositivo de films adhesivos no tóxicos empleados en la protección y consolidación del estrato pictórico: análisis de la pérdida de masa y rango de dureza". Arché. Publicación del Instituto Universitario de Restauración del Patrimonio de la Upv, 4 y 5: $132-138$

VIVAR, I., BORREGO, S.F., ALFONSO, M., OCHOA, J., RODRIGUEZ, M.E. (2016). Estudio de las condiciones ambientales en los depósitos del Archivo Nacional de la República de Cuba. Conservar Património, 24: 55-62. https://doi.org/10.13140/ RG.2.2.24663.68001

WAGNER, S. (1996). "Remoistenable tissue part II: variations on a theme". The Book and Paper Group Annual, 15: 27-28.

WHISTLER, R.L., PASCHALL, E.F. (1965). Starch: Chemistry and Technology. Academic Press eds. New York, NY.

ZAPPALÀ, A. (1990). Introduzione agliinterventi di restauro conservativo di beni culturali caracei, Ed. del Bianco, Udine: 34 . 


\section{Autor/es}

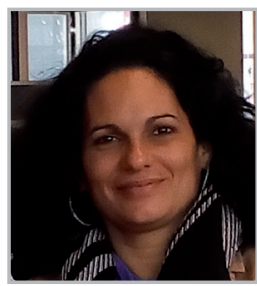

\section{Janet Díaz Navarro}

janet@arnac.cu

Laboratorio de Conservación Preventiva del Archivo Nacional de la República de Cuba

Es licenciada en Radioquímica, Master en Química Orgánica, Investigadora Agregada del Laboratorio de Conservación Preventiva del Archivo Nacional de la República de Cuba y Profesora Adjunta de la Universidad de La Habana. Se ha vinculado desde hace más de 15 años a la investigación sobre la conservación y restauración del patrimonio documental. Ha presentado trabajos en más de 20 eventos nacionales e internacionales y es autora de más 15 artículos científicos publicados en revistas nacionales e internacionales.

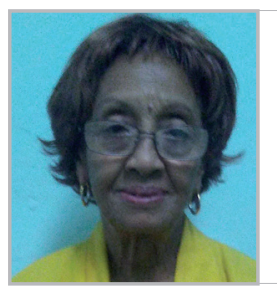

\section{Inés Baró Valle}

ines@arnac.cu

Especialista en Restauración del Archivo Nacional de la República de Cuba

Especialista en Restauración del Archivo Nacional de la República de Cuba. Vinculada a esta labor desde hace 50 años. Ha recibido múltiples cursos en el extranjero (Rusia, España, Estados Unidos). Asesora la actividad de restauración en la Red de Archivos Históricos de Cuba. Ha impartido más de 50 cursos dentro del país y en el exterior (República Dominicana, Isla Gran Caimán, México). Ha presentado trabajos en más de 30 en eventos nacionales e internacionales y ha publicado varios artículos científicos y algunos libros relacionados con la restauración de documentos siendo el más reciente "La Gestión sostenible de un laboratorio de restauración: experiencias en el Archivo Nacional de la República de Cuba". Editorial TREA, España. 2016.

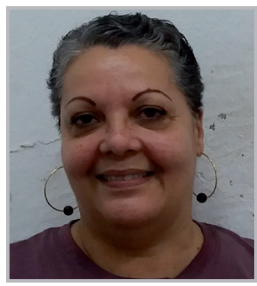

\author{
Sofía Flavia Borrego Alonso \\ sofy.borrego@gmail.com \\ Laboratorio de Conservación Preventiva \\ del Archivo Nacional de la República de \\ Cuba \\ https://orcid.org/0000-0001-8739-2577
}

Jefa del Laboratorio de Conservación Preventiva del Archivo Nacional de la República de Cuba es licenciada en Microbiología, Doctora en Ciencias Biológicas, Investigadora Titular del archivo y Profesora Titular (Universidad de La Habana y Universidad de las Artes). Investiga en las líneas de: Conservación preventiva del patrimonio documental, biodeterioro de soportes documentales y calidad micológica de ambientes interiores en archivos cubanos. Dirige varios proyectos de investigación e innovación tecnológica nacionales e internacionales y ha dirigido proyectos de colaboración con instituciones científicas y de conservación en Argentina, España, Colombia y Venezuela. Ha impartido cursos y conferencias sobre conservación preventiva del patrimonio cultural y biodeterioro de documentos en Cuba y en el extranjero (Argentina, Colombia, México, República Dominicana y Venezuela) y recientemente impartió un taller sobre Bioseguridad en los Archivos vía online para toda Iberoamérica organizado por la Asociación Latinoamericana de Archivos (ALA). Ha presentado más de 70 trabajos en eventos nacionales e internacionales y posee más de 40 publicaciones científicas en revistas nacionales e internacionales. Es revisor de múltiples revistas nacionales e internacionales y es editora de una revista inglesa (EC Microbiology).

Artículo enviado el 24/11/2020 Artículo aceptado el 18/10/2021 (@) $(\Theta \Theta$

https://doi.org/10.37558/gec.v20i.867 